\title{
Tuning the Brain-Gut Axis in Health and Disease
}

\author{
Rainbo Hultman • Michelle M. Sidor • Kafui Dzirasa
}

Published online: 15 January 2015

(C) Springer International Publishing AG 2015

\begin{abstract}
Recent breakthroughs in gut microbiome-derived technologies and therapies, coupled with the lack of invasiveness associated with them, provide attractive routes of biomarker and therapeutic development. Alongside such breakthroughs, an ever-growing body of literature indicates a strong connection between brain activity and microbial populations in the gut. The paucity of effective psychiatric therapies makes the gut microbiota/brain connection a particularly enticing field. This article reviews some of the mechanisms of connection between the gut and the brain, their potential relationship to psychiatric disorders, and the therapeutic potential that arises from them.
\end{abstract}

Keywords Microbiome $\cdot$ Gut $\cdot$ Brain $\cdot$ Probiotics $\cdot$ Enteric nervous system $\cdot$ Vagal nerve

This article is part of Topical Collection on Microbiome and Stem Cell Function

R. Hultman $\cdot$ K. Dzirasa $(\bowtie)$

Department of Psychiatry and Behavioral Sciences, Duke University

Medical Center, 361 Bryan Research Building, Box 3209, Durham,

NC 27710, USA

e-mail: kafui.dzirasa@duke.edu

\section{K. Dzirasa}

Center for Neuroengineering, Duke University Medical Center,

Durham, NC 27710, USA

\section{K. Dzirasa}

Duke Institute for Brain Sciences, Duke University Medical Center, Durham, NC 27710, USA

\section{K. Dzirasa}

Departments of Biomedical Engineering and Neurobiology, Duke

University Medical Center, Durham, NC 27710, USA

M. M. Sidor

Department of Psychiatry, University of Pittsburgh Medical School, Pittsburgh, PA 15219, USA

\section{Introduction}

Despite a rise in mental illness diagnoses, the efficacy of current treatments is low, few new treatments have made it to patients in the past several decades, and interest in developing new psychiatric drugs is waning $[1,2]$. While new technologies like deep brain stimulation are incredibly promising [3], their cost and invasiveness remain an obstacle to reaching large populations of people. One hopeful breakthrough on this front is the growing understanding of the connection between the gut microbiome and the brain. Given the ease and noninvasiveness of regulating populations of gut microbiota [4, 5], connections between the gut and brain provide a novel and groundbreaking new avenue to explore with regard to the development of new psychotherapies. Additionally, because gut microbial populations can be sampled from fecal matter, the brain-gut connection is also promising in terms of developing effective biomarkers for psychiatric disorders.

Studies demonstrating the therapeutic efficacy of gut microbial manipulations are numerous and include the following: studies in germ-free animals, infection studies, pro and antibiotic manipulation, and fecal transplant [5-8]. So much progress has been made developing probiotic therapies with regard to psychiatric disorders that an entire class of developing therapeutics has been dubbed "psychobiotics" $[4,9]$. The basic principle behind psychobiotics is that microorganisms can be introduced that either directly or through their impact on the rest of the microbiome lead to a psychotherapeutic effect. Thus far, the most successful psychobiotics have been tested as antidepressants and anxiolytics $[4,6,7,10]$. Designer probiotics have begun to be developed as well. Through this process, bacteria are genetically engineered to imbue them with specific desirable qualities such as stress tolerance prior to administering $[11,12]$. Antibiotics have tremendous potential as tools for selectively manipulating the gut microbiome $[8,13]$, and fecal transplant therapies have been proposed as 
treatments for disorders related to changes in systemic inflammation [8]. Finally, there is also evidence suggesting that dietary changes may induce gut microbial changes that regulate inflammatory response and intestinal barrier integrity, processes implicated in contributing to psychiatric disorders $[10,14,15]$.

In order to best exploit the brain-gut axis for therapeutic use, it is first necessary to understand the underlying mechanisms of connection. Mechanisms connecting the gut and brain include the following: metabolites and neurotransmitters/ neuroendocrine system, immune response, and direct synaptic projections between central and enteric nervous systems.

\section{Gut Microbiota-Derived Signaling Molecules and Neuroactive Metabolites}

Commensal gut bacteria can produce various neurotransmitters, hormones, and neuroactive metabolites, including gamma-aminobutyric acid (GABA), serotonin, histamine, melatonin, catecholamines (e.g., dopamine, norepinephrine), and nitric oxide $[16,17]$. From an evolutionary perspective, it has been proposed that these transmitters initially arose as signaling molecules for bacteria-bacteria communication [17]. As symbiotic relationships formed between the endocrine systems of bacteria and more complex host organisms, these signaling molecules became important for host-bacteria communication [17] and eventually evolved to signal internal events within the host itself [18]. For instance, certain strains of bacteria are capable of synthesizing GABA - the main inhibitory neurotransmitter in the brain-from glutamate in vitro and express a receptor-like molecule for uptake of GABA. Furthermore, both GABA and its receptor are found in gut epithelia cells $[19,20]$. Importantly, within a diseaserisk context, central GABA dysfunction in humans has been linked to diseases such as anxiety and depression [21].

Serotonin and the Kynurenine Pathway

Another important example of a critical metabolite that impacts brain function is serotonin. Although traditionally associated with central nervous system (CNS) neural transmission, the vast majority of serotonin is produced in the gut from dietary tryptophan and its availability regulated by the gut microbiome via direct and indirect mechanisms. Tryptophan is an essential amino acid and the precursor to serotonin synthesis. It is converted into 5-hydroxytryptophan (5-HTT) by the rate-limiting enzyme, tryptophan hydroxylase, which is then converted into serotonin (5-HT) via aromatic amino acid decarboxylase. Alternatively, tryptophan can be metabolized into quinolinic and kynurenic acid by 2,3-indolamine-2,3dioxygenase (IDO) along the competing kynurenine pathway. Various factors determine the ratio between serotonin synthesis and tryptophan metabolites, including interaction of the gut microbiome with these pathways [22]. Certain strains of gut bacteria can directly metabolize tryptophan and can produce both tryptophan [23] and serotonin [24]. Indirectly, particular strains can alter how tryptophan is metabolized and affect the balance between serotonin synthesis vs. kynurenine pathway production of the N-methyl-D-aspartate (NMDA) receptor modulators, kynurenic and quinolinic acid. This is particularly relevant given that increased levels of quinolinic acid have been found in both the cerebrospinal fluid (CSF) and plasma of clinically depressed patients $[25,26]$ and an increased production of kynurenic acid has been reported in schizophrenic patients [27, 28]. It is likely that this imbalance in tryptophan metabolites over serotonin synthesis modify disease risk through interaction of these metabolites with cognate receptors, thereby altering neural activity and neural circuit function.

Given that serotonin is central to a variety of physiological processes (body temperature regulation, circadian rhythms, pain sensitivity, stress response, appetite, etc.) and that altered serotonergic function has been implicated in a host of neuropsychiatric diseases [29], it is plausible that the gut microbiome may directly influence disease susceptibility via its actions on host serotonin availability.

Collectively, the ability of the microbiome to both directly and indirectly impinge on transmitter synthesis and metabolic pathways suggests that gut bacteria may influence brain function and disease risk through its actions on neurotransmitter availability.

\section{Immune Response and the Brain}

One recent hypothesis for mechanisms underlying the connection between gut microbiota and brain, particularly under stressed or other pathological circumstances, is that of "the leaky gut." The inner lining of the gut is composed of a layer of epithelial cells connected by tight junctions with a mucosal covering that form a barrier sealing off the gut contents to the rest of the body [30]. One major point of communication between gut and immune system is at the mucosal barrier of the gut, which contains dendritic cells and macrophages [30]. The balance between recognition of bacteria as commensal or pathogenic is finely tuned and regulated by Toll-like receptors (TLRs), which are responsible for inflammatory and homeostatic responses [31]. When the immune response is launched against pathogenic microbes, the subsequent release of cytokines, such as interleukin (IL)-6, IL-1, and tumor necrosis factor (TNF)- $\alpha$, can have profound impacts on brain function. For example, inflammatory responses in the hippocampus, hypothalamus, prefrontal cortex, and amygdala have been shown to lead to psychiatric disruption such as in the case of 
major depressive disorder (MDD) [32-37]. Parenthetically, antidepressant selective serotonin re-uptake inhibitors (SSRIs) have been shown to reduce levels of inflammatory cytokines [10]. Finally, stress which has been shown to trigger and exacerbate symptoms of psychiatric disorders in humans and in rodents, has also been shown to trigger inflammatory responses [10, 34].

Some evidence suggests that increased gut epithelial permeability underlies major depressive disorder and other affective disorders brought on after major or chronic stress [7]. This phenomenon, also referred to as the "leaky gut hypothesis," indicates that under certain circumstances, such as in the case of the presence of a psychosocial stressor, the tight junctions of the epithelium become more permeable, enabling the translocation of bacteria and bacterial secretions that would otherwise be prevented from escaping. Thus, the resultant effect is a systemic immune response [7, 35]. There are several studies suggesting possible mechanisms by which such increased gut permeability occurs [30]. Several specific types of bacteria appear to be associated with gut permeability, in particular, an increase in the order Bacteroidales and parallel decrease in Clostridiales have been shown to correlate with gut permeability in mice [38•]. It will be interesting to determine whether manipulations of gut populations that mediate gut leakiness and inflammation can be developed into effective therapeutics for psychiatric disorders.

\section{Connecting Central and Enteric Nervous Systems}

In addition to the impact of the gut via endocrine and immune responses, there are also direct reciprocal afferents and efferents between the gut (enteric nervous system, ENS) and the brain (CNS), providing a third level of regulation to explore and exploit. These projections fall largely into three major subcategories: vagal, spinal thoracolumbar, and spinal lumbosacral [39]. Chemical and mechanical stimuli in the gut lead to stimulation of afferents in these neurons leading ultimately to neuronal signaling at the level of the CNS [40]. Furthermore, CNS information flows to the gut primarily via motor efferents responding to hypothalamic-pituitary-adrenal axis (HPA) and digestive-related cues.

The enteric nervous system, sometimes referred to as the "second brain" because of its complexity, is composed of some 300-600 million neurons (in humans) [39, 41, 42]. These neurons are structured into complex ganglia networks throughout the stomach, intestines, esophagus, pancreas, and more [39]. They can be largely categorized as belonging to either the myenteric ganglia (part of muscular layers) or the submucosal plexus (restricted primarily to the intestines) and regulate a wide array of functions including blood flow, secretion, and motor as well as some of the immune and endocrine responses already discussed [43, 44]. Enteric neurons include intrinsic primary afferent neurons (IPANs), which respond to various mechanical and chemical triggers and have projections that are well integrated into synaptic networks of the gut - they are perhaps the most widely connected [43]. Other key neuronal components making up the circuits of the ENS include the following: excitatory motorneurons, inhibitory circular muscle motorneurons, longitudinal muscle motorneurons, ascending interneurons, descending interneurons, and secretomotor and vasomotor neurons [39, 43].

The vagus nerve appears to be responsible for a great deal of the communication between the ENS and CNS and has both sensory and motor properties. The enteric nervous system sends messages through the vagus nerve signaling changes in neuronal firing and chemical (including nutrient and hormone) as well as limited mechanical stimuli [40]. With over 40,000 axons, the vagus nerve afferent mechanisms are complex and diverse; for in-depth review of these mechanisms, see Furness J.B. et al. [39]. The vagus nerve has been shown to be particularly linked to microbiota-induced behavioral changes in several key studies [45•, 46•, 47, 48]. Interestingly, enteric neuron excitability appears to be an important component of such vagal-mediated behavioral regulation [45•], demonstrating that communication between the ENS and CNS plays a role in gut microbiota-driven CNS biology.

While it is still somewhat unclear exactly how signals that pass through the vagus nerve communicate with higher order brain function, it is clear that the hypothalamus, amygdala, locus coeruleus, hippocampus, and several forebrain regions are profoundly affected $[42,49,50]$. Additionally, the anterior insula (aINS) stands out as the most likely hub of emotional information connecting the gut with other CNS emotional processing regions, including the anterior cingulate (ACC), the orbitofrontal cortex (OFC), and the amygdala [42]. The INS has been shown to be involved in the mediation of emotional state, disgust, and social behaviors [42]. Vagal outputs make connections with nearly every component of the enteric nervous system, primarily through cholinergic excitatory pathways [44]. This pathway has been implicated in modulating appetite and gut digestive mechanics [44], providing a clear route for CNS to ENS information flow.

\section{Gut Microbiota and Brain Development}

In addition to the many routes by which the gut microbiome impacts the mature brain, there is a growing body of evidence that microbial populations have a significant impact on brain development as well $[51,52]$. Colonization of the gut by microbiota occurs early in development, during the first few minutes following birth. The environment, therefore, provides the initial source of microbiota that colonize the gut. Throughout the lifespan, both environmental and genetic 
factors influence the type and diversity of colonies that take up residence in the gut with a stable adult-like population emerging early in development. Each individual, however, contains a "unique bacterial fingerprint" [53] as a result of distinct gene $\times$ environment interactions that occur over the lifespan.

Much of what we know about the colonization of the early gut and its long-term impact on brain development and function comes from work utilizing germ-free mice. These mice are completely devoid of commensal microbiota at birth and exhibit an undeveloped immune system along with behavioral abnormalities in adulthood [54]. This includes, altered serotonergic and plasticity-related gene expression in key limbic brain regions associated with mood disorders [55, 56], altered hypothalamic-pituitary-adrenal (HPA) axis function [57], and accompanying behavioral dysfunction [52, 55-57]. Importantly, re-colonization of germ-free mice with commensal bacteria at key critical time points during early postnatal development can alter their developmental trajectory and reverse molecular and behavioral abnormalities [52, 57]. This points to a direct role for the gut microbiome in normal brain development and function. Furthermore, the dramatic influence that gut bacteria have on levels and turnover of monoamines such as serotonin and dopamine, which have been shown to play an important role in regulating neuron development, suggests a mechanism whereby such regulation is likely to take place [58-62]. Indeed, altering serotonin levels

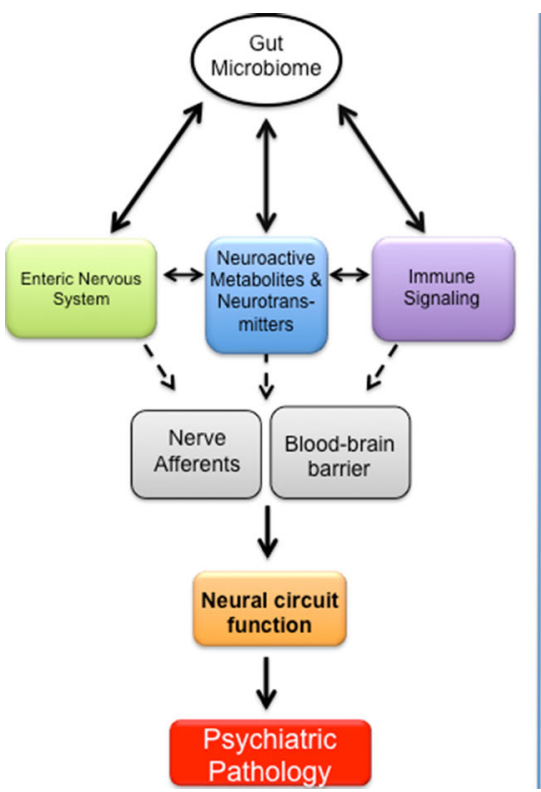

Fig. 1 A mechanistic convergence of gut microbial populations on brain function. There are many pathways by which the gut microbiome may impact neural circuit function in health and disease. Three key nodes that are described in this review are illustrated: the enteric nervous system, neuroactive metabolites and neurotransmitters, and immune signaling. It is likely these bidirectional processes and pathways converge to influence brain development and function over the lifetime. The left panel demonstrates mechanisms by which changes in gut microbial populations can result in the onset or exacerbation of psychiatric at key points during brain development in rodents increases the vulnerability to developing behavioral dysfunction in adulthood [63-66]. Finally, manipulations of gut bacteria have been shown to play a significant role in the regulation of the neurotrophin brain-derived neurotrophic factor (BDNF) [67], a well-known modulator of neuron development [68-71].

Given that a link has been identified between gut bacteria, immune response and neurodevelopmental disorders such as autism, further study into the mechanisms by which gut microbiota impact brain development are highly promising therapeutically as well $[72,73]$.

\section{Conclusions}

\section{Putting it Together-Routes to Therapeutics}

With multiple direct and indirect interactions between brain and gut physiology and several lines of evidence indicating that gut microbiota contribute to these interactions (Fig. 1), system studies that examine brain physiology, gut physiology, and gut microbiota function concurrently in the same animal will be critical to elucidating the mechanisms whereby the brain-gut axis establishes homeostasis. Ultimately, this understanding will create a novel framework to explore how changes in these systems lead to psychopathology.

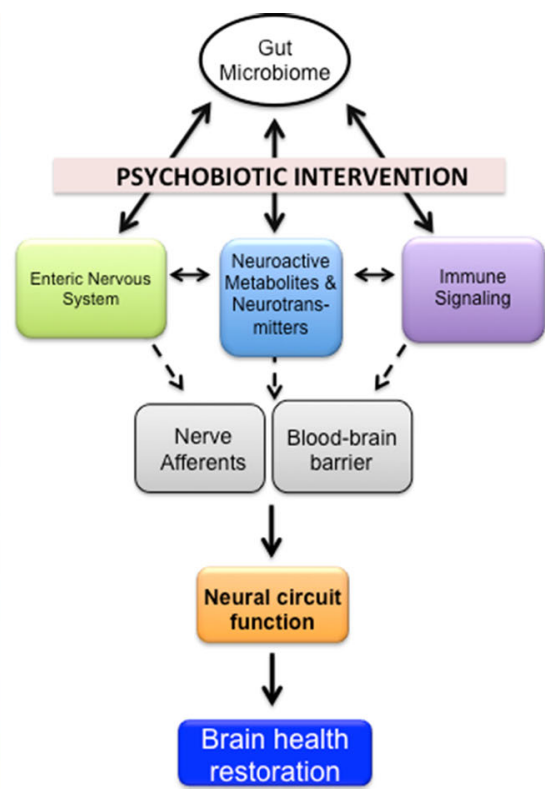

disorders. The right panel highlights that by manipulating gut bacterial populations with specific probiotics, brain health can be restored. Both the vagal nerve and the blood-brain barrier represent two proposed routes for gut-brain communication. The brain is especially vulnerable to insult during critical developmental time windows in which gut-brain signaling may be crucial for modifying disease-risk in susceptible individuals. Targeting the gut microbiome through the use of psychobiotics (as shown in the right-hand panel) may prove to be a useful therapeutic avenue for preventing or attenuating psychopathology 


\section{Compliance with Ethics Guidelines}

Conflict of Interest Rainbo Hultman, Michelle M Sidor, and Kafui Dzirasa declare that they have no conflict of interest.

Human and Animal Rights and Informed Consent This article does not contain any studies with human or animal subjects performed by any of the authors.

\section{References}

Papers of particular interest and published recently are highlighted below as:

- Of importance

1. Akil H, Brenner S, Kandel E, Kendler KS, King MC, Scolnick E, et al. Medicine. The future of psychiatric research: genomes and neural circuits. Science. 2010;327:1580-1.

2. S. E. Hyman. Psychiatric drug development: diagnosing a crisis. Cerebrum : Dana Forum Brain Sci. 2013; 5 (2013)

3. Dzirasa K, Lisanby SH. How does deep brain stimulation work? Biol Psychiatry. 2012;72:892-4. doi:10.1016/j.biopsych.2012.09. 013.

4. Wall R, Cryan JF, Ross RP, Fitzgerald GF, Dinan TG, Stanton C. Bacterial neuroactive compounds produced by psychobiotics. Adv Exp Med Biol. 2014;817:221-39. doi:10.1007/978-1-4939-08974_10.

5. Bravo JA, Julio-Pieper M, Forsythe P, Kunze W, Dinan TG, Bienenstock J, et al. Communication between gastrointestinal bacteria and the nervous system. Curr Opin Pharmacol. 2012;12:66772. doi:10.1016/j.coph.2012.09.010.

6. Tang F, Reddy BL, Saier Jr MH. Psychobiotics and their involvement in mental health. J Mol Microbiol Biotechnol. 2014;24:211-4. doi:10.1159/000366281.

7. Smythies LE, Smythies JR. Microbiota, the immune system, black moods and the brain-melancholia updated. Front Hum Neurosci. 2014;8:720. doi:10.3389/fnhum.2014.00720.

8. Borre YE, Moloney RD, Clarke G, Dinan TG, Cryan JF. The impact of microbiota on brain and behavior: mechanisms \& therapeutic potential. Adv Exp Med Biol. 2014;817:373-403. doi:10.1007/ 978-1-4939-0897-4 17

9. Dinan TG, Stanton C, Cryan JF. Psychobiotics: a novel class of psychotropic. Biol Psychiatry. 2013;74:720-6. doi:10.1016/j. biopsych.2013.05.001.

10. Berk M, Williams LJ, Jacka FN, O'Neil A, Pasco JA, Moylan S, et al. So depression is an inflammatory disease, but where does the inflammation come from? BMC Med. 2013;11:200. doi:10.1186/ 1741-7015-11-200.

11. Paton AW, Morona R, Paton JC. Designer probiotics for prevention of enteric infections. Nat Rev Microbiology. 2006;4:193-200. doi: 10.1038/nrmicro1349.

12. Sleator RD, Shortall C, Hill C. Metagenomics. Lett Appl Microbiol. 2008;47:361-6. doi:10.1111/j.1472-765X.2008.02444.x.

13. Modi SR, Collins JJ, Relman DA. Antibiotics and the gut microbiota. J Clin Invest. 2014;124:4212-8. doi:10.1172/JCI72333.

14. Alonso C, Vicario M, Pigrau M, Lobo B, Santos J. Intestinal barrier function and the brain-gut axis. Adv Exp Med Biol. 2014;817:73113. doi:10.1007/978-1-4939-0897-4 4.

15. Hullar MA, Fu BC. Diet, the gut microbiome, and epigenetics. Cancer J. 2014;20:170-5. doi:10.1097/PPO.0000000000000053.
16. Lyte M. Microbial endocrinology and the microbiota-gut-brain axis. Adv Exp Med Biol. 2014;817:3-24. doi:10.1007/978-1-49390897-4 1 .

17. Iyer LM, Aravind L, Coon SL, Klein DC, Koonin EV. Evolution of cell-cell signaling in animals: did late horizontal gene transfer from bacteria have a role? Trends Genet. 2004;TIG 20:292-9. doi:10. 1016/j.tig.2004.05.007.

18. Lyte $\mathrm{M}$. The microbial organ in the gut as a driver of homeostasis and disease. Med Hypotheses. 2010;74:634-8. doi:10.1016/j.mehy. 2009.10.025.

19. Forsythe P, Sudo N, Dinan T, Taylor VH, Bienenstock J. Mood and gut feelings. Brain Behav Immun. 2010;24:9-16. doi:10.1016/j.bbi. 2009.05.058.

20. Nakajima K, Tooyama I, Kuriyama K, Kimura H. Immunohistochemical demonstration of GABAB receptors in the rat gastrointestinal tract. Neurochem Res. 1996;21:211-5.

21. Cryan JF, Kaupmann K. Don't worry 'B' happy!: a role for GABA(B) receptors in anxiety and depression. Trends Pharmacol Sci. 2005;26:36-43. doi:10.1016/j.tips.2004.11.004.

22. O'Mahony SM, Clarke G, Borre YE, Dinan TG, Cryan JF. Serotonin, tryptophan metabolism and the brain-gut-microbiome axis. Behav Brain Res. 2014. doi:10.1016/j.bbr.2014.07.027.

23. Li G, Young KD. Indole production by the tryptophanase TnaA in Escherichia coli is determined by the amount of exogenous tryptophan. Microbiology. 2013;159:402-10. doi:10.1099/mic.0. 064139-0.

24. Lyte M. Probiotics function mechanistically as delivery vehicles for neuroactive compounds: microbial endocrinology in the design and use of probiotics. BioEssays : News Rev Mol Cell Dev Biol. 2011;33:574-81. doi:10.1002/bies.201100024.

25. Myint AM, Kim YK, Verkerk R, Scharpe S, Steinbusch H, Leonard B. Kynurenine pathway in major depression: evidence of impaired neuroprotection. J Affect Disord. 2007;98:143-51. doi:10.1016/j. jad.2006.07.013.

26. Raison CL, Dantzer R, Kelley KW, Lawson MA, Woolwine BJ, Vogt $\mathrm{G}$, et al. CSF concentrations of brain tryptophan and kynurenines during immune stimulation with IFN-alpha: relationship to CNS immune responses and depression. Mol Psychiatry. 2010;15:393-403. doi:10.1038/mp.2009.116.

27. Erhardt S, Blennow K, Nordin C, Skogh E, Lindstrom LH, Engberg G. Kynurenic acid levels are elevated in the cerebrospinal fluid of patients with schizophrenia. Neurosci Lett. 2001;313:96-8.

28. Linderholm KR, Skogh E, Olsson SK, Dahl ML, Holtze M, Engberg G, et al. Increased levels of kynurenine and kynurenic acid in the CSF of patients with schizophrenia. Schizophr Bull. 2012;38: 426-32. doi:10.1093/schbul/sbq086.

29. Lucki I. The spectrum of behaviors influenced by serotonin. Biol Psychiatry. 1998;44:151-62.

30. Smythies LE, Smythies JR. Exosomes in the gut. Front Immunol. 2014;5:104. doi:10.3389/fimmu.2014.00104.

31. Honda K, Takeda K. Regulatory mechanisms of immune responses to intestinal bacteria. Mucosal Immunol. 2009;2:187-96. doi:10. 1038/mi.2009.8.

32. Muller N. Immunology of major depression. Neuroimmunomodulation. 2014;21:123-30. doi:10.1159/ 000356540 .

33. McCusker RH, Kelley KW. Immune-neural connections: how the immune system's response to infectious agents influences behavior J Exp Biol. 2013;216:84-98. doi:10.1242/jeb.073411.

34. Lotrich FE. Inflammatory cytokine-associated depression. Brain Res. 2014. doi:10.1016/j.brainres.2014.06.032.

35. Maes M, Kubera M, Leunis JC, Berk M. Increased IgA and IgM responses against gut commensals in chronic depression: further evidence for increased bacterial translocation or leaky gut. J Affect Disord. 2012;141:55-62. doi:10.1016/j.jad.2012.02.023. 
36. Maes M, Mihaylova I, Kubera M, Ringel K. Activation of cellmediated immunity in depression: association with inflammation, melancholia, clinical staging and the fatigue and somatic symptom cluster of depression. Prog Neuropsychopharmacol Biol Psychiatry. 2012;36:169-75. doi:10.1016/j.pnpbp.2011.09.006.

37. Miller $\mathrm{AH}$, Maletic $\mathrm{V}$, Raison CL. Inflammation and its discontents: the role of cytokines in the pathophysiology of major depression. Biol Psychiatry. 2009;65:732-41. doi:10.1016/j.biopsych.2008.11. 029.

38. Schwab C, Berry D, Rauch I, Rennisch I, Ramesmayer J, Hainzl E, et al. Longitudinal study of murine microbiota activity and interactions with the host during acute inflammation and recovery. ISME J. 2014;8:1101-14. doi:10.1038/ismej.2013.223. This study monitors gut microbiome changes over time in response to acute inflammation and demonstrates correlation of specific bacteria with increased gut permeability.

39. Furness JB, Callaghan BP, Rivera LR, Cho HJ. The enteric nervous system and gastrointestinal innervation: integrated local and central control. Adv Exp Med Biol. 2014;817:39-71. doi:10.1007/978-14939-0897-4 3 .

40. Furness JB, Rivera LR, Cho HJ, Bravo DM, Callaghan B. The gut as a sensory organ. Nat Rev Gastroenterol Hepatol. 2013;10:72940. doi:10.1038/nrgastro.2013.180.

41. Gershon MD. V. Genes, lineages, and tissue interactions in the development of the enteric nervous system. Am J Physiol. 1998;275:G869-73.

42. Mayer EA. Gut feelings: the emerging biology of gut-brain communication. Nat Rev Neurosci. 2011;12:453-66. doi:10.1038/ nrn3071.

43. Costa M, Brookes SJ, Hennig GW. Anatomy and physiology of the enteric nervous system. Gut. 2000;47 Suppl 4:iv15-9. discussion iv26.

44. Forsythe P, Bienenstock J, Kunze WA. Vagal pathways for microbiome-brain-gut axis communication. Adv Exp Med Biol. 2014;817:115-33. doi:10.1007/978-1-4939-0897-4_5.

45. Bercik P, Park AJ, Sinclair D, Khoshdel A, Lu J, Huang X, et al. The anxiolytic effect of Bifidobacterium longum NCC3001 involves vagal pathways for gut-brain communication. Neurogastroenterol Motil : off J Eur Gastrointest Motil Soc. 2011;23:1132-9. doi:10.1111/j.1365-2982.2011.01796.x. This study determined a specific gut bacterium (B. longum) exerts an anxiolytic behavioral response via the vagus nerve and impacts enteric neuron excitability.

46. Bravo JA, Forsythe P, Chew MV, Escaravage E, Savignac HM, Dinan TG, et al. Ingestion of Lactobacillus strain regulates emotional behavior and central GABA receptor expression in a mouse via the vagus nerve. Proc Natl Acad Sci U S A. 2011;108:16050-5. doi:10.1073/pnas.1102999108. This study demonstrates a clear and direct vagus nerve-dependent mechanism by which a gut bacterium (L. rhamnosus) changes GABA signaling in the brain.

47. Bercik P, Verdu EF, Foster JA, Macri J, Potter M, Huang X, et al. Chronic gastrointestinal inflammation induces anxiety-like behavior and alters central nervous system biochemistry in mice. Gastroenterology. 2010;139:2102-12. doi:10.1053/j.gastro.2010. 06.063. e2101.

48. Kunze WA, Mao YK, Wang B, Huizinga JD, Ma X, Forsythe P, et al. Lactobacillus reuteri enhances excitability of colonic AH neurons by inhibiting calcium-dependent potassium channel opening. J Cell Mol Med. 2009;13:2261-70. doi:10.1111/j.1582-4934.2009. 00686.x.

49. Berntson GG, Sarter M, Cacioppo JT. Ascending visceral regulation of cortical affective information processing. Eur J Neurosci. 2003;18:2103-9.

50. Craig AD. An ascending general homeostatic afferent pathway originating in lamina I. Prog Brain Res. 1996;107:225-42.
51. Al-Asmakh M, Anuar F, Zadjali F, Rafter J, Pettersson S. Gut microbial communities modulating brain development and function. Gut Microbes. 2012;3:366-73. doi:10.4161/gmic.21287.

52. Diaz Heijtz R, Wang S, Anuar F, Qian Y, Bjorkholm B, Samuelsson $\mathrm{A}$, et al. Normal gut microbiota modulates brain development and behavior. Proc Natl Acad Sci U S A. 2011;108:3047-52. doi:10. 1073/pnas.1010529108.

53. Cryan JF, O'Mahony SM. The microbiome-gut-brain axis: from bowel to behavior. Neurogastroenterol Motil : Off J Eur Gastrointest Motil Soc. 2011;23:187-92. doi:10.1111/j.13652982.2010.01664.x.

54. Foster JA, McVey Neufeld KA. Gut-brain axis: how the microbiome influences anxiety and depression. Trends Neurosci. 2013;36:305-12. doi:10.1016/j.tins.2013.01.005.

55. Neufeld KA, Kang N, Bienenstock J, Foster JA. Effects of intestinal microbiota on anxiety-like behavior. Commun Integr Biol. 2011;4: 492-4. doi:10.4161/cib.4.4.15702.

56. Clarke G, Grenham S, Scully P, Fitzgerald P, Moloney RD, Shanahan F, et al. The microbiome-gut-brain axis during early life regulates the hippocampal serotonergic system in a sex-dependent manner. Mol Psychiatry. 2013;18:666-73. doi:10.1038/mp.2012. 77.

57. Sudo N, Chida Y, Aiba Y, Sonoda J, Oyama N, Yu XN, et al. Postnatal microbial colonization programs the hypothalamicpituitary-adrenal system for stress response in mice. J Physiol. 2004;558:263-75. doi:10.1113/jphysiol.2004.063388.

58. Fricker AD, Rios C, Devi LA, Gomes I. Serotonin receptor activation leads to neurite outgrowth and neuronal survival. Brain Res Mol Brain Res. 2005;138:228-35. doi:10.1016/j.molbrainres.2005. 04.016.

59. Ponimaskin E, Voyno-Yasenetskaya T, Richter DW, Schachner M, Dityatev A. Morphogenic signaling in neurons via neurotransmitter receptors and small GTPases. Mol Neurobiol. 2007;35:278-87.

60. Mattson MP. Neurotransmitters in the regulation of neuronal cytoarchitecture. Brain Res. 1988;472:179-212.

61. B. S. Reinoso, A. S. Undie, P. Levitt. Dopamine receptors mediate differential morphological effects on cerebral cortical neurons in vitro. J Neurosci Res. 1996; 43, 439-453;published online EpubFeb 15 (10.1002/(SICI)1097-4547(19960215) 43:4<439::AID-JNR5>3.0.CO;2-G [pii]10.1002/(SICI)10974547(19960215)43:4\&1t;439::AID-JNR5\&gt;3.0.CO;2-G).

62. Prokosch V, Panagis L, Volk GF, Dermon C, Thanos S. Alpha2adrenergic receptors and their core involvement in the process of axonal growth in retinal explants. Invest Ophthalmol Vis Sci. 2010;51:6688-99. doi:10.1167/iovs.09-4835.

63. Gross C, Zhuang X, Stark K, Ramboz S, Oosting R, Kirby L, et al. Serotonin1A receptor acts during development to establish normal anxiety-like behaviour in the adult. Nature. 2002;416:396-400. doi: $10.1038 / 416396 a$.

64. Ansorge MS, Zhou M, Lira A, Hen R, Gingrich JA. Earlylife blockade of the 5-HT transporter alters emotional behavior in adult mice. Science. 2004;306:879-81. doi:10.1126/ science. 1101678 .

65. Lo Iacono L, Gross C. Alpha-Ca2+/calmodulin-dependent protein kinase II contributes to the developmental programming of anxiety in serotonin receptor 1A knock-out mice. J Neurosci. 2008;28: 6250-7. doi:10.1523/JNEUROSCI.5219-07.2008.

66. Vinkers $\mathrm{CH}$, Oosting RS, van Bogaert MJ, Olivier B, Groenink L. Early-life blockade of 5-HT(1A) receptors alters adult anxiety behavior and benzodiazepine sensitivity. Biol Psychiatry. 2010;67: 309-16. doi:10.1016/j.biopsych.2009.08.013.

67. Bercik P, Denou E, Collins J, Jackson W, Lu J, Jury J, et al. The intestinal microbiota affect central levels of brainderived neurotropic factor and behavior in mice. 
Gastroenterology. 2011;141:599-609. doi:10.1053/j.gastro. 2011.04.052. 609 e591-593.

68. Binder DK, Scharfman HE. Brain-derived neurotrophic factor. Growth Factors. 2004;22:123-31.

69. Davies AM, Thoenen H, Barde YA. The response of chick sensory neurons to brain-derived neurotrophic factor. J Neurosci. 1986;6: 1897-904.

70. Zagrebelsky M, Korte M. Form follows function: BDNF and its involvement in sculpting the function and structure of synapses. Neuropharmacology. 2014;76 Pt C:628-38. doi:10.1016/j. neuropharm.2013.05.029.
71. Park H, Poo MM. Neurotrophin regulation of neural circuit development and function. Nat Rev Neurosci. 2013;14:7-23. doi:10. $1038 /$ nrn3379.

72. Hsiao EY, McBride SW, Hsien S, Sharon G, Hyde ER, McCue T, et al. Microbiota modulate behavioral and physiological abnormalities associated with neurodevelopmental disorders. Cell. 2013;155:1451-63. doi:10.1016/j.cell.2013. 11.024 .

73. Rook GA, Raison CL, Lowry CA. Microbiota, immunoregulatory old friends and psychiatric disorders. Adv Exp Med Biol. 2014;817: 319-56. doi:10.1007/978-1-4939-0897-4_15. 